\title{
Theoretical Aspects of Spinodal Decomposition in $\mathrm{Fe}-\mathrm{C}$
}

\begin{abstract}
B. KIM, J. SIETSMA, and M.J. SANTOFIMIA
Carbon redistribution is known to occur during room temperature aging of $\mathrm{Fe}-\mathrm{C}$ martensite. One of the proposed mechanisms in the literature by which carbon redistributes is spinodal decomposition, a thermodynamically driven reaction in which the alloy undergoes separation into carbon-rich and carbon-poor regions, giving rise to modulations in carbon concentration. Despite the substantial experimental evidence supporting the occurrence of spinodal decomposition in $\mathrm{Fe}-\mathrm{C}$, its theoretical formulation requires attention. In the present study, a theoretical framework based on the regular solution model is built for evaluating the thermodynamics of the Fe-C system, with particular emphasis on the interstitial nature of carbon atoms within the ferrite lattice. Assuming a defect-free lattice, the model explains a miscibility gap in the Fe-C system. The limitations of the current model are critically evaluated.
\end{abstract}

https://doi.org/10.1007/s11661-018-5094-1

(C) The Author(s) 2019

\section{INTRODUCTION}

THERE is vast experimental evidence that shows carbon redistribution during the room-temperature aging of $\mathrm{Fe}-\mathrm{C}$ martensite, giving rise to fine modulations in carbon content across martensite. Two main processes have been proposed in the literature to account for this observation: (i) carbon segregation into the vicinity of defects, ${ }^{[1]}$ and (ii) spinodal decomposition. ${ }^{[2]}$ Both processes lead to a reduction in the free energy of the supersaturated solid solution in ferrite. However, a clear distinction must be made between the two. As discussed in previous studies by the current authors, ${ }^{[3,4]}$ both defect segregation and spinodal decomposition are strongly related to the thermodynamic description of the ferrite phase.

Carbon segregation to defects is a well-understood phenomenon, and has been extensively modeled, particularly in the context of strain aging. ${ }^{[5,6]}$ On the other hand, spinodal decomposition in $\mathrm{Fe}-\mathrm{C}$ systems still remains unclear. With the advances in experimental techniques, the topic has recently drawn significant interest from the scientific community. ${ }^{[7-9]}$ Most of the spinodal decomposition literature has been focused on

B. KIM is with the Department of Engineering, Lancaster University, Lancaster LA1 4YW, UK and also with the Department of Materials Science and Engineering, Delft University of Technology, Mekelweg 2, 2628 CD, Delft, The Netherlands. Contact e-mail: b.kimlee@lancaster.ac.uk J. SIETSMA and M. J. SANTOFIMIA are with the Department of Materials Science and Engineering, Delft University of Technology

Manuscript submitted July 20, 2017.

Article published online January 3, 2019 the experimental work on Fe-Ni-C systems, as exemplified by References 2, 4, 7 and 8 However, evidence for spinodal decomposition occurring in the iron-carbon binary system is scarce. Ren and Wang ${ }^{[10]}$ presented a theoretical analysis of the spinodal decomposition in $\mathrm{Fe}-\mathrm{C}$ systems, and experimentally showed the occurrence of spinodal decomposition in a Fe-1.83wt. pct $\mathrm{C}$ system $^{[11]}$ by means of electron microscopy. More recently, Naraghi et al. ${ }^{[12]}$ aimed to incorporate the carbon-ordering processes occurring during martensite aging in the $\mathrm{Fe}-\mathrm{C}$ system into ThermoCalc. In agreement with the earlier postulation by Taylor et al., ${ }^{[2]}$ Naraghi et al. ${ }^{[12]}$ showed that for $\mathrm{Fe}-\mathrm{C}$, the overall system's free energy was the highest for disordered dissolution of carbon in BCC. The energy was then shown to decrease successively by Zener-ordering and spinodal decomposition.

It is emphasized that spinodal decomposition explicitly requires the presence of a miscibility gap caused by the characteristic double-minima Gibbs free energy curve (discussed in Section II). Within the spinodal, a system is regarded as being thermodynamically unstable with respect to compositional fluctuations. Therefore, in order to lower the system's free energy, the system decomposes into a mixture of two stable compositions on either side of the miscibility gap. ${ }^{[13]}$ In the case of $\mathrm{Fe}-\mathrm{C}$, spinodal decomposition would give rise to a mixture of carbon-rich and carbon-poor regions. However, there are certain thermodynamic aspects of the $\mathrm{Fe}-\mathrm{C}$ system that require further evaluation in order to ascertain the presence of a miscibility gap. The aim of the current study is to build the theoretical framework for modeling spinodal decomposition applied to Fe-C binary systems. First, a brief theoretical background will 
be given in order to introduce the relevant thermodynamic properties. The study by Ren and Wang ${ }^{[10]}$ will be revisited since parts of their theoretical formulation require critical evaluation. Finally, the current approach will be presented based on a regular solution model applied to an interstitial solution.

\section{BACKGROUND TO SPINODAL DECOMPOSITION}

Consider a binary system A-B that produces a random solution mix of $\mathrm{A}$ - and $\mathrm{B}$-atoms. In the presence of a miscibility gap, the system undergoes a separation of B-poor and B-rich regions, which gives rise to compositional modulations in the material. In spinodal decomposition, there are two aspects that need to be considered:

- Chemical spinodal: the process results in the minimization of the free energy contribution of the chemical atomic interaction,

- Coherent spinodal: the spinodal resulting from the incorporation of the counterbalancing strain energy arising from the lattice mismatch owing to the difference in solute concentration between the uniform matrix and the modulated structure.

It is noted that throughout the present study, only the thermodynamic aspects will be considered.

\section{A. The A-B System Using a Substitutional Model Approach}

Following the regular solution model, the overall free energy for an A-B binary system with a given crystal structure at a given $T$ is given by

$$
G_{\mathrm{AB}}=x_{\mathrm{A}} G_{\mathrm{A}}^{\circ}+x_{\mathrm{B}} G_{\mathrm{B}}^{\circ}+\Delta H_{\mathrm{AB}}-T \Delta S_{\mathrm{AB}},
$$

where $x_{\mathrm{A}}$ and $x_{\mathrm{B}}$ are the molar fractions of $\mathrm{A}$ and $\mathrm{B}$, respectively. $G_{\mathrm{A}}^{\circ}$ and $G_{\mathrm{B}}^{\circ}$ are the molar Gibbs free energy of pure $\mathrm{A}$ and pure $\mathrm{B}$ having the given crystal structure, respectively. The term $\left(\Delta H_{\mathrm{AB}}-T \Delta S_{\mathrm{AB}}\right)$ corresponds to the free energy of mixing, $\Delta G_{\mathrm{mix}}$, where $\Delta H_{\mathrm{AB}}$ and $\Delta S_{\mathrm{AB}}$ are the molar enthalpy and entropy of mixing, respectively, and $T$ is the temperature. Modeling $\Delta G_{\operatorname{mix}}$ is fundamental in predicting the miscibility gap properties of the system, as this is the term that determines the overall stability of the mixture A-B.

As is done in the classical regular solution model, the system's free energy is assumed to be composed of atomic pair interactions. The solution A-B will contain interatomic bonds of A-A, B-B, and A-B types. The enthalpy term $\Delta H_{\mathrm{AB}}$ for a regular solution containing a mole of atoms with fractions $x_{\mathrm{A}}$ and $x_{\mathrm{B}}$ of $\mathrm{A}$ and $\mathrm{B}$ is given by ${ }^{[14]}$

$$
\begin{gathered}
\Delta H_{\mathrm{AB}}=\Omega x_{\mathrm{A}} x_{\mathrm{B}}, \\
\Omega=N_{A} z \varepsilon,
\end{gathered}
$$

where $N_{A}$ is Avogadro's constant, $z$ is the number of nearest neighbors per atom, and $\varepsilon=\varepsilon_{\mathrm{AB}}-\frac{1}{2}\left(\varepsilon_{\mathrm{AA}}+\varepsilon_{\mathrm{BB}}\right)$, where $\varepsilon_{\mathrm{AA}}, \varepsilon_{\mathrm{BB}}$, and $\varepsilon_{\mathrm{AB}}$ are the bond energies for A-A-, $\mathrm{B}-\mathrm{B}-$, and A-B-type bonds, respectively.

The entropy term $\Delta S_{\mathrm{AB}}$ is determined by the number of ways in which atoms can be arranged at atom sites, and, assuming a random distribution of atoms, is given by

$$
\Delta S_{\mathrm{AB}}=-R\left(x_{\mathrm{A}} \ln x_{\mathrm{A}}+x_{\mathrm{B}} \ln x_{\mathrm{B}}\right) .
$$

Given that in a substitutional solid solution, $x_{\mathrm{A}}+x_{\mathrm{B}}=1$, the following is obtained:

$$
\begin{aligned}
G_{\mathrm{AB}}= & \left(1-x_{\mathrm{B}}\right) G_{\mathrm{A}}^{\circ}+x_{\mathrm{B}} G_{\mathrm{B}}^{\circ}+\Omega\left(1-x_{\mathrm{B}}\right) x_{\mathrm{B}} \\
& +R T\left[\left(1-x_{\mathrm{B}}\right) \ln \left(1-x_{\mathrm{B}}\right)+x_{\mathrm{B}} \ln x_{\mathrm{B}}\right] .
\end{aligned}
$$

If $\Delta H_{\mathrm{AB}}>0$ (positive enthalpy of mixing), a chemical spinodal can occur between the fractions $x_{1}$ and $x_{2}$, determined by the second derivative of $G_{\mathrm{AB}}$ with respect to $x_{\mathrm{B}}$ :

$$
\left(\frac{\mathrm{d}^{2} \mathrm{G}_{\mathrm{AB}}}{\mathrm{dx}_{\mathrm{B}}^{2}}\right)_{x_{1}}=\left(\frac{\mathrm{d}^{2} \mathrm{G}_{\mathrm{AB}}}{\mathrm{dx}_{\mathrm{B}}^{2}}\right)_{x_{2}}=0
$$

Figure 1 illustrates the basic thermodynamics of spinodal decomposition. For the purpose of illustration consider a hypothetical system where $\Omega=10 \mathrm{~kJ} / \mathrm{mol}$, giving rise to positive enthalpy of mixing $\left(\Delta H_{\mathrm{AB}}>0\right)$.

At higher temperatures (Figure 1(a)), $T \Delta S_{\mathrm{AB}}>\Delta H_{\mathrm{AB}}$ for all compositions and so $\Delta G_{\mathrm{AB}}$ has a positive curvature for all compositions. At lower temperatures (Figure 1(b)), in the range of composition $x_{1}=0.2$ and $x_{2}=0.8$ molar fractions, $\Delta G_{\mathrm{AB}}$ develops a negative curvature. The solution for $x_{1}$ and $x_{2}$ in Eq. [5] as a function of temperature is represented in Figure 1(c), where the miscibility gap in A-B is shown, where it shows that at $T>601 \mathrm{~K}$, there is no spinodal decomposition for the chosen value $\Omega=10 \mathrm{~kJ} / \mathrm{mol}$.

It is necessary to emphasize the fact that the above example is developed for a substitutional system, where $\mathrm{B}$ atoms take up lattice sites of $\mathrm{A}$ atoms. In the case of the $\mathrm{Fe}-\mathrm{C}$ solid solution, the carbon atoms are located in a different sublattice. We will now model the Fe-C system, taking two sublattices into account.

\section{B. Interstitial Model Approach as Opposed to a Substitutional View of the Fe-C System.}

In a previous article in this journal, Ren and Wang presented a thermodynamic model for spinodal decomposition occurring in Fe-C martensite. ${ }^{[10]}$ The purpose of this section is to recapitulate the thermodynamic framework used in their study, and to discuss some fundamental challenges in their model. In order to distinguish the study in the literature from our current study, all thermodynamic expressions derived from the study by Ren and Wang ${ }^{[10]}$ are clearly marked by subscript "RW." Although a working mathematical model was built, there are a few issues that require further discussion. The authors in Reference 10 have based their model on the fact that the molar fractions of carbon and of iron sum to unity, i.e., $x_{\mathrm{C}}+x_{\mathrm{Fe}}=1$, where $x_{i}$ is the molar fraction of element $i$. The 
expression for free energy of mixing obtained by Ren and Wang, using $x_{\mathrm{C}}+x_{\mathrm{Fe}}=1$, was

$$
\Delta G_{\mathrm{RW}}=I_{0}\left(1-x_{\mathrm{C}}\right)^{2} x_{\mathrm{C}}+R T\left(x_{\mathrm{C}} \ln x_{\mathrm{C}}+x_{\mathrm{Fe}} \ln x_{\mathrm{Fe}}+x_{\mathrm{C}} \ln 2\right),
$$

where by applying the second differential of $G$ with respect to $x_{\mathrm{C}}$, Ren and Wang presented the following expression:

$$
\frac{\mathrm{d}^{2} \mathrm{G}_{\mathrm{RW}}}{\mathrm{dx}_{\mathrm{C}}^{2}}=-2 I_{0}\left(2-3 x_{\mathrm{C}}\right)+\frac{R T}{x_{\mathrm{C}}},
$$

in which $I_{0}$ is the heat of solution of carbon in ferrite, having a numerical value of $8.4 \times 10^{4} \mathrm{~J} \mathrm{~mol}^{-1}$. In their original study, the source of this value for $I_{0}$ is not stated.

By combining Eqs. [5] and [7], a quadratic equation is obtained in the form $\left[6 I_{0} x_{\mathrm{C}}^{2}-4 I_{0} x_{\mathrm{C}}+R T=0\right]$. Solving this quadratic equation, the miscibility gap shown in Figure 2(a) was obtained by Ren and Wang. The coherent spinodal will be commented in Section IV-A. Following the expression $x_{\mathrm{C}}+x_{\mathrm{Fe}}=1$ stated in
Reference 10, the equivalent graph using carbon mass fraction, $w_{\mathrm{C}}$ is shown in Figure 2(b), given that

$$
w_{\mathrm{C}}=\frac{x_{\mathrm{C}} M_{\mathrm{C}}}{x_{\mathrm{C}} M_{\mathrm{C}}+\left(1-x_{\mathrm{C}}\right) M_{\mathrm{Fe}}},
$$

where $M_{i}$ is the atomic mass of element $i$.

Following the thermodynamic formulation of Reference 10 , a miscibility gap is expected up to $\sim 1200 \mathrm{~K}$, and at $300 \mathrm{~K}$ the coherent spinodal gives carbon concentrations for the carbon-poor and carbon-rich regions of $\sim 0.01$ and $7.74 \mathrm{wt}$. pct, respectively. This does not match well to the experimental observations typically made in the literature. ${ }^{2]}$

Furthermore, since the authors in Reference 10 stated that $x_{\mathrm{C}}+x_{\mathrm{Fe}}=1$, the substitution of $x_{\mathrm{Fe}}=1-x_{\mathrm{C}}$ throughout Eq. [6] leads to

$$
\begin{aligned}
\Delta G_{\mathrm{RW}}= & I_{0}\left(1-x_{\mathrm{C}}\right)^{2} x_{\mathrm{C}}+R T\left[x_{\mathrm{C}} \ln x_{\mathrm{C}}\right. \\
& \left.+\left(1-x_{\mathrm{C}}\right) \ln \left(1-x_{\mathrm{C}}\right)+x_{\mathrm{C}} \ln 2\right] .
\end{aligned}
$$

The difference between Eqs. [6] and [9] will lead to a significant mathematical difference in the second differential of $G_{\mathrm{RW}}$ in Eq. [9] with respect to $x_{\mathrm{C}}$ :

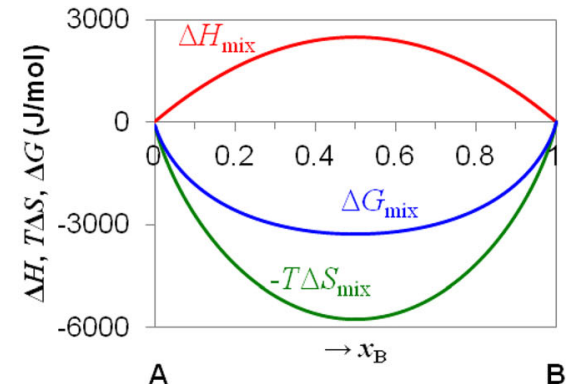

$\Omega>0$, high $T$

$(\Omega=10 \mathrm{~kJ} / \mathrm{mol}, T=1000 \mathrm{~K})$

(a)

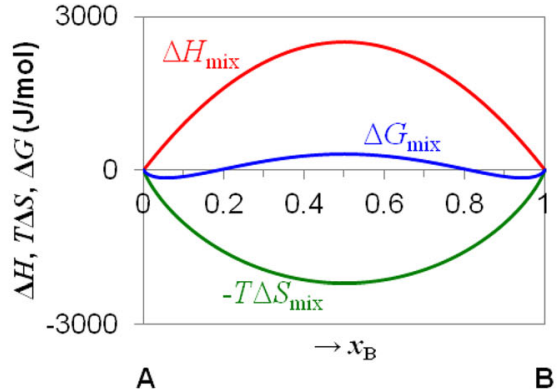

$\Omega>0$, low $T$

$(\Omega=10 \mathrm{~kJ} / \mathrm{mol}, T=380 \mathrm{~K})$

(b)

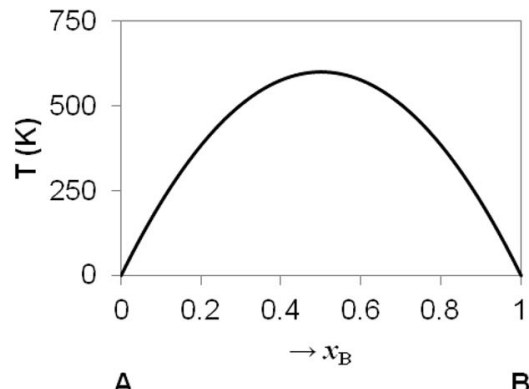

B

(c)

Fig. 1-Regular solution model for the hypothetical system A-B where the mixture leads to a strongly positive enthalpy of mixing. (a) At high temperatures $(T=1000 \mathrm{~K})$, there is no miscibility gap, but $(b)$ at lower temperatures $(T=380 \mathrm{~K})$, a miscibility gap is present. $(c)$ Spinodal shown in the A-B phase diagram based on Eq. [5].

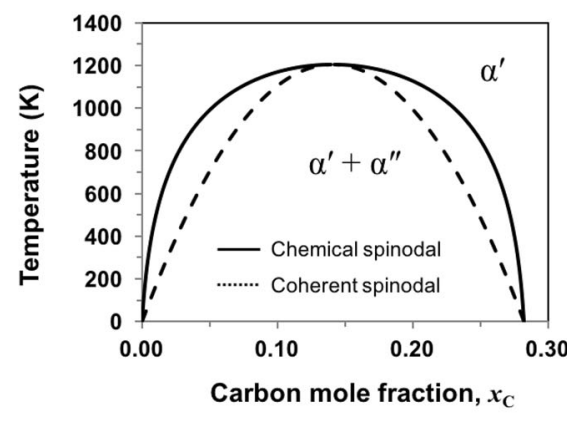

(a)

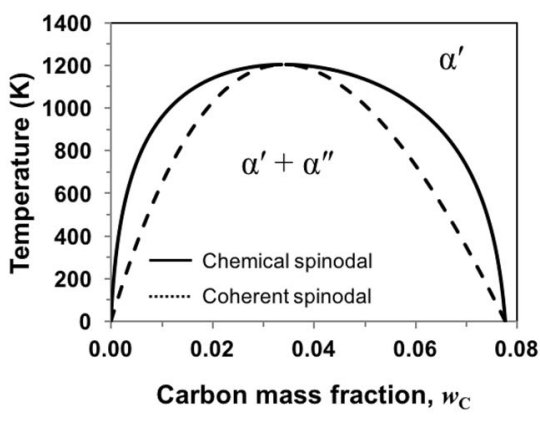

(b)

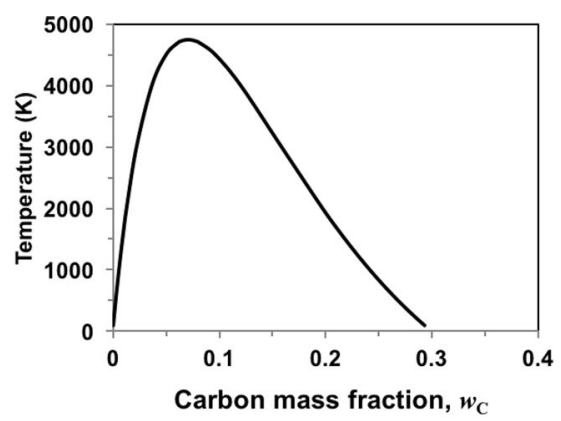

(c)

Fig. 2-Reproduction of the spinodal curves derived from the model proposed by Ren and Wang. The chemical spinodal curves have been obtained using Eq. [7]. For the expressions used in determining the coherent spinodal curves, the readers may refer to the original reference. ${ }^{[10]}$ Spinodal curves shown in $(a)$ carbon mole fraction $\left(x_{\mathrm{C}}\right)$, and $(b)$ carbon mass fraction $\left(w_{\mathrm{C}}\right)$. $(c)$ Miscibility gap derived using Eq. [10], shown only for $0 \leq w_{\mathrm{C}} \leq 0.3$. 


$$
\frac{\mathrm{d}^{2} \mathrm{G}_{\mathrm{RW}}}{\mathrm{dx}_{\mathrm{C}}^{2}}=I_{0}\left(-4+6 x_{\mathrm{C}}\right)+R T\left[\frac{1}{x_{\mathrm{C}}}+\frac{1}{\left(1-x_{\mathrm{C}}\right)}\right] .
$$

Following the same procedure as above, the combination of Eqs. [5] and [10] produces a cubic equation in the form of $\left[I_{0}\left(-4 x_{\mathrm{C}}+10 x_{\mathrm{C}}^{2}-6 x_{\mathrm{C}}^{3}\right)+R T=0\right]$. Solving this cubic equation, a different miscibility gap is obtained (expressed in terms of carbon mass fraction), as shown in Figure 2(c), where Ren and Wang's value $I_{0}=8.4 \times 10^{4} \mathrm{~J} \mathrm{~mol}^{-1}$ is used. Comparing Figures 2(b) and (c), although the same thermodynamic description of the system is applied, different miscibility gaps are obtained. For instance, at $T=300 \mathrm{~K}$, the model obtained from Eq. [6] gives molar fractions $x_{\mathrm{C}}=0.004$ and 0.278 for the carbon-poor and carbon-rich concentrations, respectively. These values differ from those obtained from Eq. [9], which yields $x_{\mathrm{C}}=0.008$ and 0.645 for carbon-poor and carbon-rich regions, respectively.

Therefore, a more robust model is needed in order to avoid such discrepancy in results. For the current approach, based on the framework presented in Section II, we build step-by-step a regular solution model for describing the $\mathrm{Fe}-\mathrm{C}$ system.

\section{THE INTERSTITIAL MODEL}

The description of the $\mathrm{Fe}-\mathrm{C}$ system used for the current model is shown in Figure 3(a), where it is shown that iron atoms occupy the substitutional lattice, which generates an interstitial sublattice at which carbon atoms and vacant sites are situated.

In the current model, it is assumed that the substitutional lattice is fully occupied by iron atoms. The effect of lattice defects in the Fe-C system is addressed in Section IV-B in detail.

The interstitial sublattice is composed of vacant sites and carbon atoms. The total number of interstitial sites, $N_{i}$, is given by the number of carbon atoms and vacant sites: $N_{i}=N_{\mathrm{C}}+N_{\mathrm{Va}}$. Therefore, the site fraction concept is introduced, where

$$
s_{\mathrm{C}}=\frac{N_{\mathrm{C}}}{N_{i}} ; s_{\mathrm{Va}}=\frac{N_{\mathrm{Va}}}{N_{i}} .
$$

$N_{i}$ is intrinsic to the iron lattice, and is given by $N_{i}=\beta N_{\mathrm{Fe}}$, where $\beta$ is the ratio of interstitial sites to the number of substitutional sites. For the BCC lattice, $\beta=$ 3 according to Zener ${ }^{[15]}$ and $\beta=2$ according to Ren and Wang. ${ }^{[10]}$ This will be discussed in Section III-C.

The outline of the free energy graph for the described system is shown in Figure 3(b). $s_{\mathrm{C}}=0$ represents the scenario where all interstitial sublattice sites are vacant, and no carbon atoms are present, essentially referring to a state of pure iron. The notation used to describe the free energy of both lattices in this case is $G_{\mathrm{Fe}: \mathrm{Va}}^{\circ}$. On the other hand, $s_{\mathrm{C}}=1$ refers to a situation where all interstitial sublattice sites are occupied by carbon atoms, where the free energy is given by $G_{\mathrm{Fe}: \mathrm{C}}^{\circ}$.

Describing the thermodynamics of the $\mathrm{Fe}-\mathrm{C}$ system is complicated by the interstitial nature of the carbon atoms. Hillert ${ }^{[16]}$ proposed an interstitial solutes model that takes into account the concept of excluded sites, where an interstitial solute atom blocks its neighboring sites from being occupied. However, his approach was that of an ideal solution, where the enthalpy of mixing was neglected. Our approach is to build a thermodynamic description that captures the $\mathrm{Fe}-\mathrm{C}$ system based on the regular solution model: assigning the enthalpy to the pairwise nearest neighbor interactions between atoms, and calculating the entropy on the basis of random positioning of atoms on the lattice, taking carbon-carbon interactions into account.

\section{A. Free Energy of Mixing}

Building up from Figure 3(b), the molar free energy of the overall system is described as

$$
G F e C=\left(1-s_{\mathrm{C}}\right) G_{\mathrm{Fe}: \mathrm{Va}}^{\circ}+s_{\mathrm{C}} G_{\mathrm{Fe}: \mathrm{C}}^{\circ}+\Delta G_{(\mathrm{Fe}: \mathrm{Va}, \mathrm{C})}^{\circ},
$$

where $\Delta G_{(\mathrm{Fe}: \mathrm{Va}, \mathrm{C})}^{\circ}$ is the free energy of mixing of carbon and vacant sites within the iron substitutional lattice. By definition, $\quad \Delta G_{(\mathrm{Fe}: \mathrm{Va}, \mathrm{C})}^{\circ}=\Delta H_{(\mathrm{Fe}: \mathrm{Va}, \mathrm{C})}^{\circ}-T \Delta S_{(\mathrm{Fe}: \mathrm{Va}, \mathrm{C})}^{\circ}$, where $T$ is the temperature and $\Delta H_{(\mathrm{Fe}: \mathrm{Va}, \mathrm{C})}^{\circ}$ and $\Delta S_{(\mathrm{Fe}: \mathrm{Va}, \mathrm{C})}^{\circ}$ are the enthalpy and entropy of the Fe-C mixture, respectively.

Applying Eq. [2] for the case of Fe-C interstitial solution, the following expression for enthalpy per mole of iron atoms follows from the regular solution scheme:

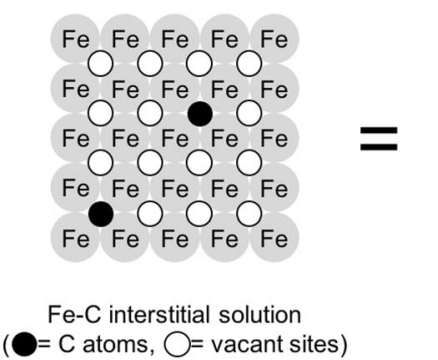

$=\mathrm{C}$ atoms, $\mathrm{O}=$ vacant sites)

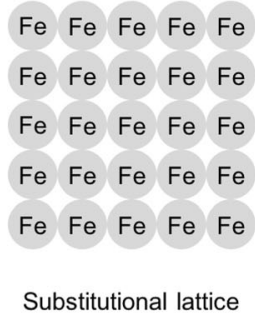

(a)

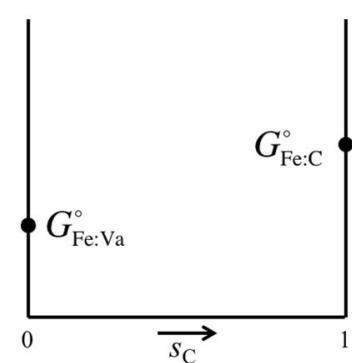

(b)

Fig. 3-(a) Representation of the interstitial model, where the system consists of a substitutional lattice composed of Fe atoms, and an interstitial sublattice which can contain carbon atoms. (b) Schematics of the free energy diagram where $s_{\mathrm{C}}$ represents the site fraction at the interstitial sublattice occupied by carbon atoms. 


$$
\Delta H_{(\mathrm{Fe}: \mathrm{Va}, \mathrm{C})}=\Omega_{(\mathrm{Fe}: \mathrm{Va}, \mathrm{C})}\left[s_{\mathrm{C}}\left(1-s_{\mathrm{C}}\right)\right],
$$

where $\Omega_{(\mathrm{Fe}: \mathrm{Va}, \mathrm{C})}$ represents the interaction parameter between the main substitutional lattice and the interstitial lattice, and is defined by the following:

$$
\begin{aligned}
& \Omega_{(\mathrm{Fe}: \mathrm{Va}, \mathrm{C})}=z_{\mathrm{is}} N_{i}\left[\varepsilon_{(\mathrm{Fe}: \mathrm{VaC})}-\frac{1}{2}\left(\varepsilon_{(\mathrm{Fe}: \mathrm{VaVa})}+\varepsilon_{(\mathrm{Fe}: \mathrm{CC})}\right)\right], \\
& =z_{\mathrm{is}} \beta N_{A}\left[\varepsilon_{(\mathrm{Fe}: \mathrm{VaC})}-\frac{1}{2}\left(\varepsilon_{(\mathrm{Fe}: \mathrm{VaVa})}+\varepsilon_{(\mathrm{Fe}: \mathrm{CC})}\right)\right], \quad
\end{aligned}
$$

where $z_{\text {is }}$ is the number of nearest neighbors that an interstitial element has on the substitutional lattice, $N_{i}$ was previously defined as the total number of interstitial sites, and the various $\varepsilon$ terms represent the bond energies for the various bond pairs: the terms $\varepsilon_{(\mathrm{Fe}: \mathrm{CC})}$, $\varepsilon_{(\mathrm{Fe}: \mathrm{VaVa})}$ and $\varepsilon_{(\mathrm{Fe}: \mathrm{VaC})}$ represent the $\mathrm{C}-\mathrm{C}, \mathrm{Va}-\mathrm{Va}$, and Va-C bonds, respectively, found on the interstitial lattice within the Fe substitutional lattice. Since vacancies will not mutually interact within the iron substitutional lattice, the $\varepsilon_{(\mathrm{Fe}: \mathrm{VaVa})}$ term is considered to be zero. The molar change in enthalpy is thus given by

$$
\Delta H_{(\mathrm{Fe}: \mathrm{Va}, \mathrm{C})}=z_{\mathrm{is}} \beta N_{A}\left(\varepsilon_{(\mathrm{Fe}: \mathrm{VaC})}-\frac{1}{2} \varepsilon_{(\mathrm{Fe}: \mathrm{CC})}\right)\left[s_{\mathrm{C}}\left(1-s_{\mathrm{C}}\right)\right] .
$$

The interstitial lattice, composed of carbon atoms and vacancies, contributes to the molar entropy as follows:

$$
\begin{aligned}
& \Delta S_{(\mathrm{Fe}: \mathrm{Va}, \mathrm{C})}=-k N_{i}\left[s_{\mathrm{C}} \ln s_{\mathrm{C}}+\left(1-s_{\mathrm{C}}\right) \ln \left(1-s_{\mathrm{C}}\right)\right], \\
& =-k \beta N_{A}\left[s_{\mathrm{C}} \ln s_{\mathrm{C}}+\left(1-s_{\mathrm{C}}\right) \ln \left(1-s_{\mathrm{C}}\right)\right] .
\end{aligned}
$$

This is equivalent to

$$
\Delta S_{(\mathrm{Fe}: \mathrm{Va}, \mathrm{C})}=-R \beta\left[s_{\mathrm{C}} \ln s_{\mathrm{C}}+\left(1-s_{\mathrm{C}}\right) \ln \left(1-s_{\mathrm{C}}\right)\right] .
$$

Adding the enthalpy and entropy terms, the molar free energy of the overall system is given by

$$
\begin{aligned}
G_{\mathrm{FeC}}= & \left(1-s_{\mathrm{C}}\right) G_{\mathrm{Fe}: \mathrm{Va}}^{\circ}+s_{\mathrm{C}} G_{\mathrm{Fe}: \mathrm{C}}^{\circ} \\
& +z_{\mathrm{is}} \beta N_{A}\left(\varepsilon_{(\mathrm{Fe}: \mathrm{VaC})}-\frac{1}{2} \varepsilon_{(\mathrm{Fe}: \mathrm{CC})}\right)\left[s_{\mathrm{C}}\left(1-s_{\mathrm{C}}\right)\right] \\
& +R T \beta\left[s_{\mathrm{C}} \ln s_{\mathrm{C}}+\left(1-s_{\mathrm{C}}\right) \ln \left(1-s_{\mathrm{C}}\right)\right] .
\end{aligned}
$$

In order to numerically evaluate the miscibility gap, the terms $z_{\text {is }}, \beta$, and the difference between $\varepsilon_{(\mathrm{Fe}: \mathrm{VaC})}$ and $\varepsilon_{(\mathrm{Fe}: \mathrm{CC})}$ are to be established. These will be discussed in the next sections.

\section{B. Nearest Neighbors}

In $\mathrm{BCC}$, the available interstitial sites are classified into octahedral and tetrahedral interstitial sites (OIS and TIS, respectively), where the former is known to be the favorable one for the location of carbon atoms. ${ }^{[18]}$ The OIS are shown in Figure 4(a), where these can be further categorized into 'a,' 'b,' and 'c' sites depending on the direction in which they distort the lattice, i.e., in the $[100]_{\alpha},[010]_{\alpha}$ or $[001]_{\alpha}$ directions, respectively. Each carbon atom occupying an octahedral interstitial site has six nearest-neighboring iron atoms, thus $z_{\text {is }}=6$.

\section{C. $\beta$ and Bond Energies}

As previously mentioned, $\beta$ is the ratio of interstitial atoms to the number of iron atoms. If all octahedral sites are considered, then $\beta$ would have a value of 3 . Nevertheless, in carbon supersaturated martensite, it has been reported that one type, for which we take 'c,' $\mathrm{xxxOIS}$ are preferentially occupied, thus giving rise to tetragonality in ferrite, where the ferrite cell is stretched along the $[001]_{\alpha}$ direction. If all 'c' sites were to be potentially occupied by carbon atoms, $\beta=1$. However, as it will become clearer in the following that, $\beta$ will depend on the configuration of possible locations for carbon atoms.

For obtaining information on the interaction bonds between the substitutional and interstitial lattice, the literature on Density Functional Theory (DFT) ${ }^{[17,19-22]}$ can provide numerical values. It is important to note that most of the Fe-C DFT literature studies are based on systems containing a single carbon atom within an iron supercell. The study by Domain et al. ${ }^{[17]}$ will be referenced throughout, as in their study, the effect of a second carbon atom in the system is also studied, and thus information regarding the $\mathrm{C}-\mathrm{C}$ interaction within the iron lattice is obtained.

The study by Domain et al. ${ }^{[17]}$ reports the formation enthalpy of a system where a carbon atom binds at an octahedral site, $\Delta H_{f}^{\mathrm{C}}\left(\mathrm{Fe}_{N} \mathrm{C}\right)$. This is given by

$$
\Delta H_{f}^{\mathrm{C}}\left(\mathrm{Fe}_{\mathrm{N}} \mathrm{C}\right)=\mathrm{E}\left(\mathrm{Fe}_{\mathrm{N}} \mathrm{C}\right)-\mathrm{NE}(\mathrm{Fe})-\mathrm{E}\left(\mathrm{C}_{\text {isolated }}\right) .
$$

where $N$ is the number of iron atoms within the supercell, $E(\mathrm{Fe})$ is the reference energy of pure $\mathrm{BCC}$ iron, $E\left(\mathrm{Fe}_{N} \mathrm{C}\right)$ is the energy of a system containing a single carbon atom within the $\mathrm{BCC}$ iron, and $E\left(\mathrm{C}_{\text {isolated }}\right)$ is the energy of a single carbon atom alone in a large supercell. It is worth noting that since the calculations in Reference 17 already incorporate the changes in the electronic structure and magnetic moment within the ferromagnetic supercell in the presence of interstitial carbon, the current model does not consider magnetic effects separately. 


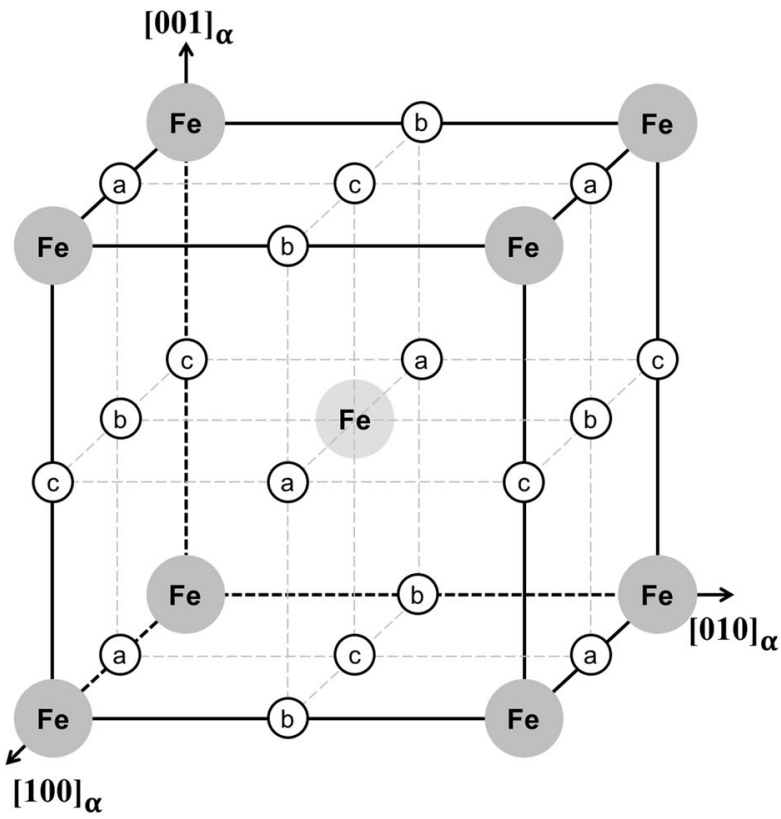

(a)

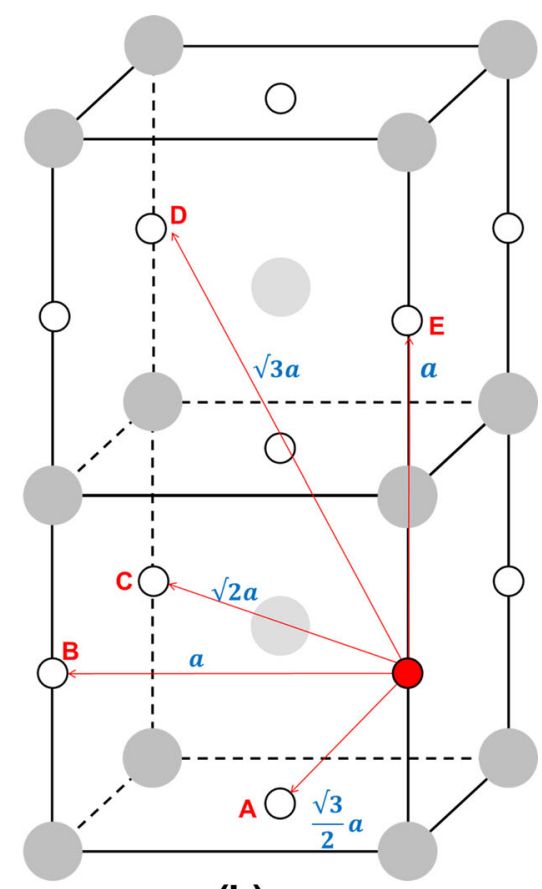

(b)

Fig. 4 - (a) Illustration of the octahedral interstitial sites (OIS) within $\alpha$-Fe with the respective classification, and (b) representation of only the 'c' OIS in ferrite, labeled as sites A-E, after Domain et al. ${ }^{[17]}$

Table I. $\quad \varepsilon_{(\mathrm{Fe}: \mathrm{CC})}$ Values Based on the C-C Binding Energy in $\alpha$-Fe Depending on the Relative Location and Distance (in Terms of Lattice Parameter a) Between Two Interstitial Carbon Atoms (Data Taken for the 54 Fe Atoms System, from Domain et al. ${ }^{[17]}$ )

\begin{tabular}{lcc}
\hline & Distance & Binding Energy, $\varepsilon_{(\mathrm{Fe}: \mathrm{CC})}(e V)$ \\
\hline Site A & $\frac{\sqrt{3}}{2} a$ & -0.42 \\
Site B & $a$ & -0.17 \\
Site C & $\sqrt{2} a$ & -0.20 \\
Site D & $\sqrt{3} a$ & -0.09 \\
Site E & $a$ & -2.28 \\
\hline
\end{tabular}

The negative values for the binding energy represent the repulsive nature of the $\mathrm{C}-\mathrm{C}$ bonds.

The enthalpy reported for the $54 \mathrm{Fe}$ atoms supercell in Reference 17 is $10.71 \mathrm{eV}$ at $0 \mathrm{~K}$ for the entire system. Therefore, $\varepsilon_{(\mathrm{Fe}: \mathrm{VaC})}=0.19 \mathrm{eV}$ per atom, equivalent to $3.1 \times 10^{-20} \mathrm{~J}$ per atom.

Furthermore, in the study by Domain et al., ${ }^{[17]}$ it is seen that a repulsive interaction exists between carbon atoms and that the magnitude of the repulsion depends on their relative positions and the distance between the two carbon atoms. In general, the further apart the two carbon atoms are, the smaller the repulsive energy. In Figure 4(b), two adjacent BCC unit cells are shown, where only one set of the OIS has been indicated. The red atom in the lower unit cell represents the first interstitial carbon atom. For the second interstitial carbon atom, a series of possible interstitial sites have been proposed by Domain et al. ${ }^{[17]}$ From their original study, only those corresponding to the same set of OIS sites are currently considered, labeled as sites A-E. The magnitude of the binding energies between the red carbon atom and a carbon atom at the possible sites are summarized in Table I. It is seen that the strongest repulsion takes place when the second carbon atom is placed in site $\mathrm{E}$, i.e., along the same axis in the adjacent unit cell, whereas the repulsion is minimized in site D, i.e., when the atoms are furthest apart with the second atom placed diagonally from the first atom.

In order to assess each type of carbon-carbon interaction individually, the repeating 'unit cell' must be carefully chosen in order to avoid introducing additional types of interaction in the system. This is illustrated in Figure 5. Let us consider carbon-carbon interaction type C (see also Figure 4(b)), where the carbon atoms are placed at distance $\sqrt{2} a$. If the building block shown in Figure 5(a) was to be the 'unit cell,' the lattice will contain carbon-carbon interactions of type D and E, Figure 5(b). To overcome this, a layer of unfilled carbon atoms is introduced under the building block in Figure 5(a). The resulting 'unit cell' is given in Figure 5(c). This will ensure that no other types of interactions are present in the lattice within the distances included in the types A-E. The carbon-carbon interaction is assumed negligible at distances greater than $2 a$, in line with the trend observed in Table I.

Following the same procedure outlined in Figure 5 for the other interactions of type A, B, D, and E, it is found that all 'unit cells' consist of a 16 Fe supercell structure containing a maximum number of two carbon atoms. Following the definition that $\beta$ is the ratio of interstitial sites to the number of substitutional sites, $\beta$ is $1 / 8$. 


\section{Free Energy of Mixing}

Based on Eqs. [15], [17], Table I, and the procedure shown in Figure 5, the enthalpy, entropy, and free energy of mixing plots of the $\mathrm{Fe}-\mathrm{C}$ mixture at $300 \mathrm{~K}$ are shown in Figure 6 for the five types of carbon-carbon interactions. The different values for $\varepsilon_{(\mathrm{Fe}: \mathrm{CC})}$ have been given for sites A-E shown in Table I. Full curves for all configurations except for $\mathrm{E}$ are shown in Figure 6, as configuration $\mathrm{E}$ is unlikely to occur given the large repulsive force between carbon atoms. It is seen that while the enthalpy of mixing is positive, the entropy contribution at $300 \mathrm{~K}$ is comparatively low. Hence, the free energy of mixing remains positive in practically the entire composition range. In order to calculate the chemical spinodal for the system, the second derivative of $G$ with respect to $s_{\mathrm{C}}$ of Eq. [18] is to be equal to zero:

$$
\frac{\mathrm{d}^{2} \mathrm{G}}{\mathrm{ds}_{\mathrm{C}}^{2}}=-2 z_{\mathrm{is}} \beta N_{A}\left(\varepsilon_{(\mathrm{Fe}: \mathrm{VaC})}-\frac{1}{2} \varepsilon_{(\mathrm{Fe}: \mathrm{CC})}\right)+\frac{R T \beta}{s_{\mathrm{C}}\left(1-s_{\mathrm{C}}\right)}=0
$$

where the maximum possible number of carbon atom is given by $\beta$, the actual carbon concentration contained within the supercell is determined by its site fraction, $s_{\mathrm{C}}$. Therefore, the carbon weight fraction in the system is determined by

$$
w_{\mathrm{C}}=\frac{\beta s_{\mathrm{C}} M_{\mathrm{C}}}{\beta s_{\mathrm{C}} M_{\mathrm{C}}+M_{\mathrm{Fe}}},
$$

where $M_{i}$ is the molar mass of element $i$.

Solving Eq. [20], the chemical spinodal is shown as a function of carbon weight fraction in Figure 7.

\section{DISCUSSION}

As shown in Figure 6, the addition of carbon within the interstitial lattice leads to an increase in the enthalpy of mixing. The enthalpy of mixing is further increased by the repulsive carbon-carbon interaction. As shown in Figure 7, this gives rise to a large miscibility gap that is seen to be stable up to $10000 \mathrm{~K}$. It must be noted that in Section III, only the chemical spinodal has been modeled. The coherent spinodal will be discussed in this section.

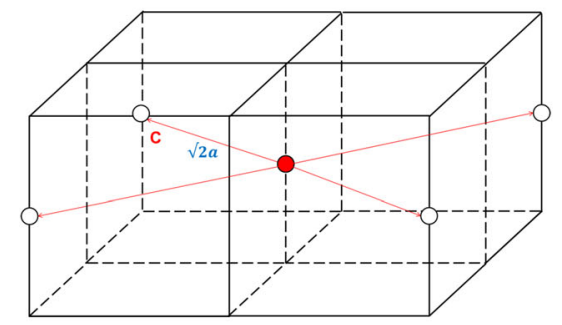

(a)

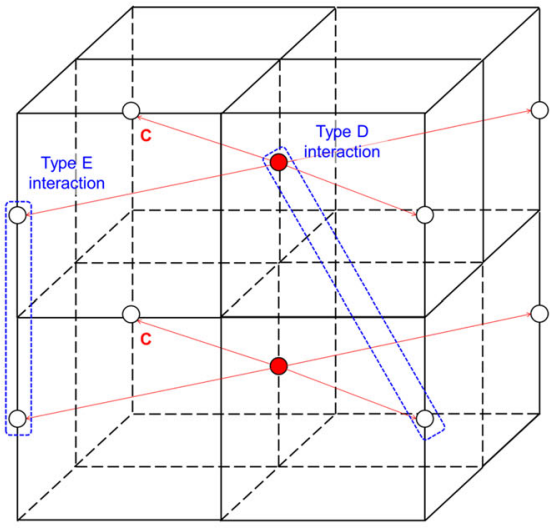

(b)

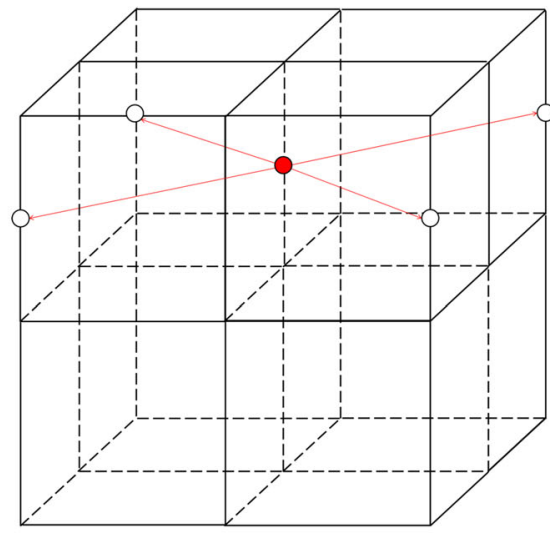

(c)

Fig. 5- (a) Representation of the type C interaction in a supercell consisting of four BCC unit cells ( 8 Fe), where carbon atoms are $\sqrt{2} a$ apart (iron atoms and unoccupied carbon sites have not been represented for clarity). (b) When the $8 \mathrm{Fe}$ supercell in (a) is stacked, additional carbon-carbon interactions of type $\mathrm{D}$ and $\mathrm{E}$ will be introduced. (c) $16 \mathrm{Fe}$ supercell proposed in order to isolate type $\mathrm{C}$ carbon-carbon interaction, by introducing a layer of four BCC unit cells containing no carbon atoms.

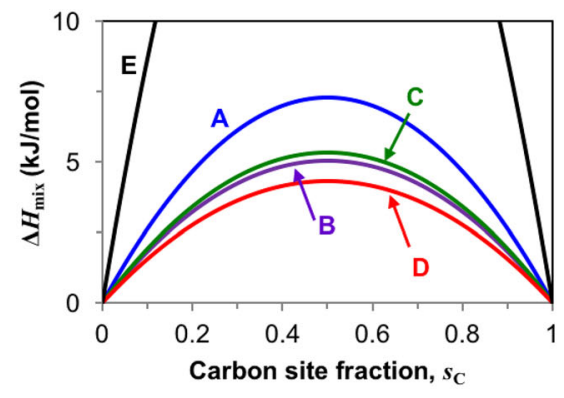

(a)

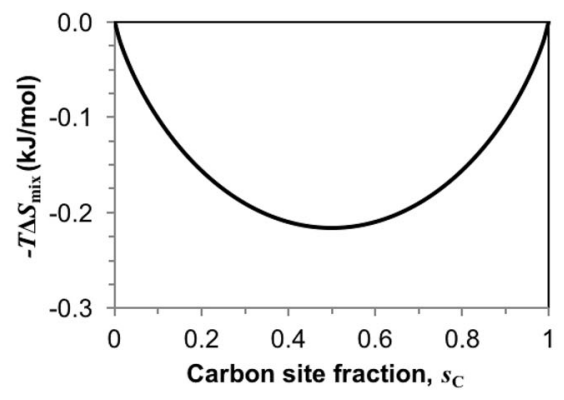

(b)

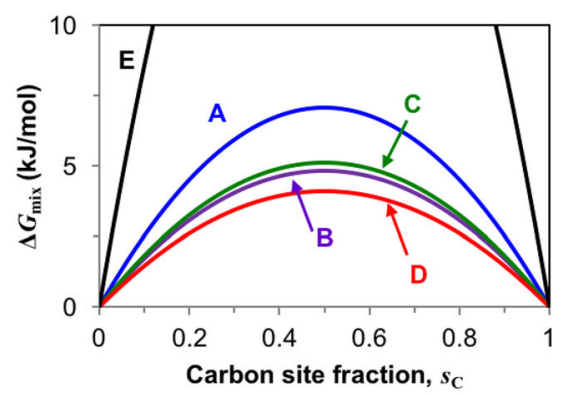

(c)

Fig. 6-Results from the Fe-C model proposed showing (a) enthalpy of mixing, (b) entropy of mixing at $300 \mathrm{~K}$, and (c) free energy of mixing at $300 \mathrm{~K}$ for the different $\varepsilon_{\mathrm{Fe}: \mathrm{CC}}$ values in octahedral interstitial sites A-E. 


\section{A. Coherent Spinodal}

Hilliard $^{[23]}$ showed that for evaluating coherent spinodal decomposition, the criterion to be used is

$$
\frac{\mathrm{d}^{2} \mathrm{G}}{\mathrm{ds}_{\mathrm{C}}^{2}}+2 \eta^{2} Y \leq 0
$$

where the term $2 \eta^{2} Y$ accounts for the elastic strain energy arising due to the density difference between the carbon-rich and carbon-poor regions. $Y$ is an elastic constant, and the density effect of concentration variations is given by the parameter $\eta$, defined as

$$
\eta=\left(\frac{1}{a_{0}}\right)\left(\frac{\delta a}{\delta x_{\mathrm{C}}}\right) \text {. }
$$

Applied to the current study, $a_{0}$ is the lattice parameter of pure BCC iron and the term $\frac{\delta a}{\delta x_{\mathrm{C}}}$ accounts for the change in iron's lattice parameter due to a change in carbon concentration.

Incorporating the expression for $\frac{\mathrm{d}^{2} \mathrm{G}}{\mathrm{ds}^{2}}$ from Eq. [20] into Eq. [22] shows that the units do not balance between the chemical spinodal (units $\mathrm{J} \mathrm{mol}^{-1}$ or $\left.\mathrm{N} \mathrm{m} \mathrm{mol}{ }^{-1}\right)$ and $2 \eta^{2} Y\left(\mathrm{~N} \mathrm{~m}^{-2}\right)$. In order to balance the units, a molar volume term, $V_{m}$ is introduced in the elastic strain energy term. This way, both terms have units of $\mathrm{J} \mathrm{mol}^{-1}$. Therefore, the coherent spinodal is obtained by

$$
\frac{\mathrm{d}^{2} \mathrm{G}}{\mathrm{ds}_{\mathrm{C}}^{2}}+2 \eta^{2} Y V_{m}=0
$$

The parameter $\eta$ accounts for the increase in lattice parameter as carbon atoms are added into solid solution. In the $\mathrm{Fe}-\mathrm{C}$ system, the addition of carbon to the 'c' interstitial sites has anisotropic effects, giving rise to tetragonality in the unit cell, as carbon redistribution occurs. At room temperature, the lattice parameters $a$ and $c$ are given by ${ }^{[24]}$

$$
\begin{aligned}
& a=0.28664 \mathrm{~nm}-(0.028 \mathrm{~nm}) \mathrm{x}_{\mathrm{C}}, \\
& c=0.28664 \mathrm{~nm}+(0.256 \mathrm{~nm}) \mathrm{x}_{\mathrm{C}},
\end{aligned}
$$

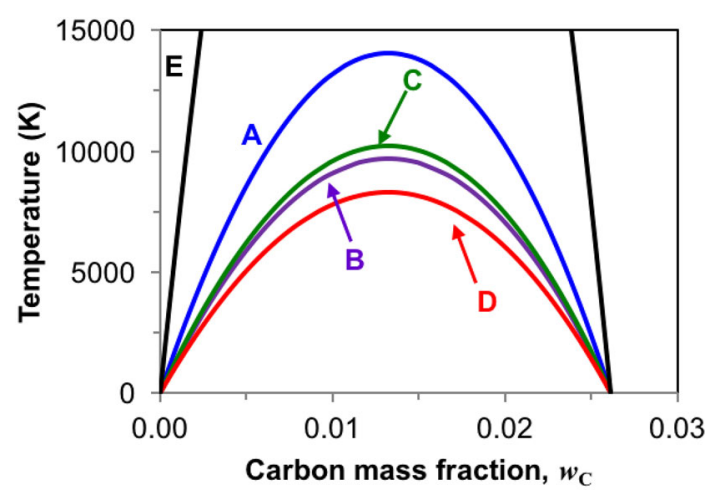

Fig. 7-Chemical spinodal of the Fe-C system for different $\varepsilon_{\mathrm{Fe}: \mathrm{CC}}$ values in octahedral interstitial sites $\mathrm{A}-\mathrm{E}$, where $w C$ has been calculated based on Eq. [21]. where $0.28664 \mathrm{~nm}$ is the lattice parameter of pure BCC iron $\left(a_{0}\right), a$ and $c$ are the lattice parameters in tetragonal ferrite, and $x_{\mathrm{C}}$ is the atomic fraction of carbon in ferrite solid solution.

The average linear expansion due to carbon in solid solution can be given by $a=0.28664 \mathrm{~nm}+\theta \mathrm{x}_{\mathrm{C}}$, where $\theta$ is the equivalent lattice expansion coefficient. $\theta$ is determined from

$$
\begin{aligned}
\left(0.28664 \mathrm{~nm}+\theta \mathrm{x}_{\mathrm{C}}\right)^{3}= & {\left[0.28664 \mathrm{~nm}-(0.028 \mathrm{~nm}) \mathrm{x}_{\mathrm{C}}\right]^{2} } \\
& {\left[0.28664 \mathrm{~nm}+(0.256 \mathrm{~nm}) \mathrm{x}_{\mathrm{C}}\right] . }
\end{aligned}
$$

A fit of this expression yields $\theta \approx 6.7 \times 10^{-2} \mathrm{~nm}$, for $x_{\mathrm{C}}$ below 0.1. ${ }^{[10]}$ Therefore, $\eta$ in Eq. [23] has a value of 0.23 .

The molar volume of iron is calculated according to

$$
V_{m}=N_{A} V_{\text {atomic }}=N_{A} \frac{a_{0}^{3}}{2},
$$

yielding $V_{m}=7.1 \times 10^{-6} \mathrm{~m}^{3} \mathrm{~mol}^{-1}$.

The elastic constant for spinodal decomposition, $Y$, is reported to be $280 \mathrm{GPa}^{[25]}$ This value has been reported for an isotropic cubic system.

Following the same procedure as in Eq. [20] but including the strain energy term $2 \eta^{2} Y V_{m}$, some coherent spinodal curves are calculated and shown in Figure 8. $\eta=0.23$ is too large to allow coherent spinodal decomposition. Instead, values which were orders of magnitude smaller yield a coherent spinodal inside the chemical spinodal.

Using the derived value $\eta=0.23$ gives a strong counterbalancing strain term to the chemical spinodal. Possible uncertainties in the determined $\eta$ value come from the fact that the carbon-rich ferrite cell is approximated to a cubic form, where in reality, the presence of carbon may lead to tetragonality in the lattice. Furthermore, the value of $Y$ is taken from an isotropic cubic form of ferrite. A more realistic approach would be to incorporate tetragonality aspects owing to carbon redistribution during spinodal decomposition, and use plane-specific $Y$ values for tetragonal martensite. Nevertheless, the difference between the calculated $(0.23)$ and the minimum possible $(\sim 0.08)$ values of $\eta$ is almost threefold. Such difference is regarded to be too large to

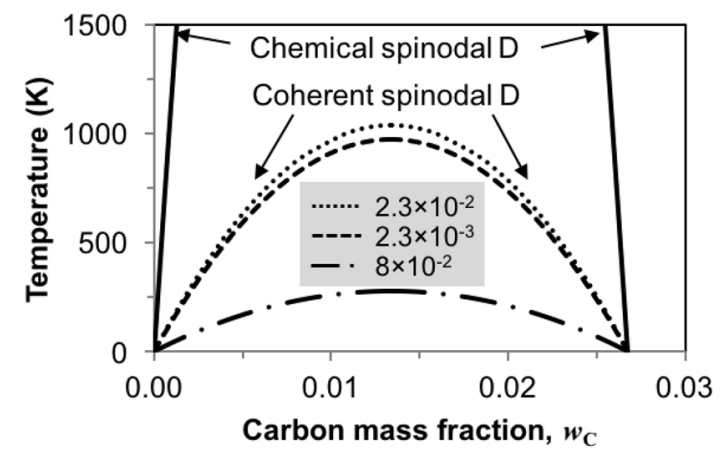

Fig. 8- Coherent spinodal for the chemical spinodal curve D, where different values of $\eta$ have been evaluated. 
be accounted in justifying the assumptions used in the current approach to model the strain contribution.

Furthermore, in comparison with the spinodal decomposition literature, the carbon-rich product is typically described as $\alpha^{\prime \prime}-\mathrm{Fe}_{16} \mathrm{C}_{2}$, where the supercell structure consisting of eight $\mathrm{BCC}$ unit cells is shown in Figure 9. The $\alpha^{\prime \prime}-\mathrm{Fe}_{16} \mathrm{C}_{2}$ product yields a carbon mass fraction of 0.026 , consistent with $\beta=1 / 8$.

This value is equal to the chemical spinodal obtained in Figure 7. This comes as no surprise, since the 'unit cells' considered are supercells containing 16 iron atoms and two carbon atoms. In fact, Figure 9 is actually the 'unit cell' of interaction $\mathrm{D}$, where the distance between neighboring carbon atoms are either $2 a$ or $\sqrt{ } 3 a$. According to the results by Domain et al., ${ }^{[17]} \mathrm{C}-\mathrm{C}$ interaction $\mathrm{D}$ results in the minimum binding energy between carbon atoms. Therefore, it becomes evident that the designated $\mathrm{Fe}_{16} \mathrm{C}_{2}$ structure results in the configuration that enables the carbon atoms to minimize their interaction energy. Modeling the coherent spinodal remains a challenge for the $\mathrm{Fe}-\mathrm{C}$ system, since the addition of carbon will transform the cubic cell into tetragonal, hence anisotropy must be considered when evaluating the $2 \eta^{2} Y V_{m}$ term.

\section{B. Vacancies in the Substitutional Lattice}

In the current model, it is assumed that the main substitutional lattice consists of a perfect crystal composed of Fe atoms only. However, this is not realistic as defects are always present, and hence a more complete picture is obtained when considering Fe-Va occupancy in the substitutional lattice, and C-Va occupancy in the

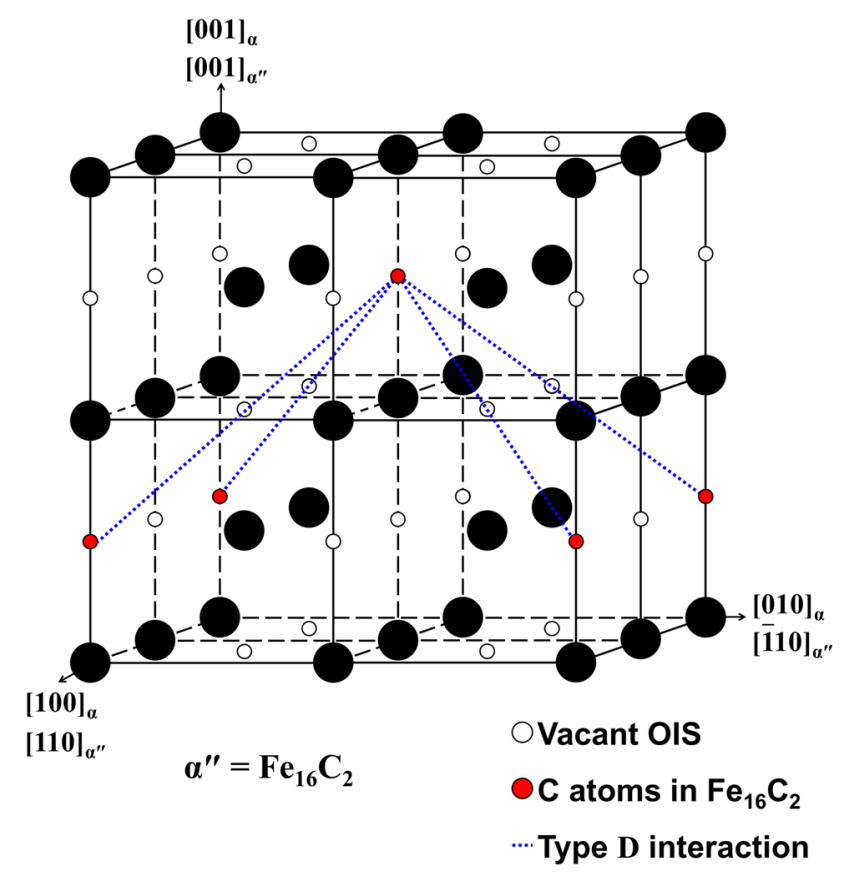

Fig. 9- The $\alpha^{\prime \prime}-\mathrm{Fe}_{16} \mathrm{C}_{2}$ structure proposed in the literature for the carbon-rich product as a result of spinodal decomposition, adapted from Ref. [18]. interstitial sublattice. The vacancy fraction $x_{v}$ in the substitutional lattice is calculated from

$$
x_{v}=\exp \left(\frac{-Q_{\mathrm{v}}}{k T}\right),
$$

where $Q_{\mathrm{v}}$ is the energy associated with the vacancy formation.

Given that $Q_{\mathrm{v}}$ is $1.4 \mathrm{eV}$ in $\mathrm{BCC}$ iron, ${ }^{[26]}$ at $300 \mathrm{~K}$, the fraction of vacancies to lattice sites in pure iron is $\sim 2.4 \times 10^{-31}$. Although this value is low, it will increase significantly in quenched specimens from austenite, since the vacancy concentration at austenitization temperature is much higher, e.g., $\sim 2.2 \times 10^{-8}$ at $1200 \mathrm{~K}$. Moreover, quenching introduces further defects in the microstructure, as martensite is characterized by its high dislocation density.

The significance of having vacant sites and other defects in the substitutional lattice is that they provide additional carbon trapping sites. Carbon-substitutional vacancy interactions have been studied in the literature, e.g., References 21,27 , and 28 where the binding energy between the interstitial carbon and the substitutional vacancy is in the range of $0.4-0.75 \mathrm{eV} .^{[27,28]}$ In particular, the study by Först et al. ${ }^{[21]}$ shows that as the number of vacant sites within the BCC lattice increases, the interaction between carbon atoms and lattice defect sites becomes dominant over the Fe-interstitial site interaction. The authors in Reference 21 have proposed that a vacant site in the substitutional lattice can bind up to two interstitial atoms. This implies that whereas in a perfect lattice, a repulsive $\mathrm{C}-\mathrm{C}$ force exists, as a vacancy is introduced, an attractive interaction builds up between the two interstitials and the vacancy.

Based on the literature findings and the current model, it appears that spinodal decomposition is limited to very specific situations. In the unlikely event of a defect-free substitutional lattice composed entirely of iron, the process of spinodal decomposition becomes dominant. Under such circumstance, the free energy of the $\mathrm{Fe}-\mathrm{C}$ system may only be reduced by the process of spinodal decomposition, by separating into carbon-rich and carbon-poor regions. The redistribution of the carbon atoms into the $\mathrm{Fe}_{16} \mathrm{C}_{2}$ supercell structure provides the lowest energy state by providing the carbon configuration that minimizes the $\mathrm{C}-\mathrm{C}$ repulsion.

However, as soon as defects are introduced in the substitutional lattice, the carbon atoms will have the possibility to remain either at regular OIS, or bind to defect sites. This line of reasoning is supported by the experimental work carried out earlier by the authors. ${ }^{[4]}$ The carbon redistribution during the room-temperature aging of two $\mathrm{Fe}-\mathrm{Ni}-\mathrm{C}$ alloys was studied in the context of spinodal decomposition $v s$ carbon segregation to defects. In the experimental work, the quenched and aged alloys were characterized using atom probe tomography and synchrotron radiation X-ray diffraction, but there was no evidence for the presence of the $\mathrm{Fe}_{16} \mathrm{C}_{2}$ structure. Instead, it was argued that carbon segregation to defects was more likely to have occurred, given the expected high dislocation density following the quenching process. 
The theoretical framework presented in the current paper gives a comprehensive thermodynamic description of $\mathrm{Fe}-\mathrm{C}$. The present study provides a fundamental basis for building up more complex processes occurring in aged martensite, as discussed above.

\section{CONCLUSIONS}

A thermodynamic framework has been developed for the $\mathrm{Fe}-\mathrm{C}$ system based on the regular solution model, and taking into consideration the interstitial nature of the carbon interstitial atoms. The chemical spinodal was seen to strongly depend on the carbon configuration within the iron lattice. Various carbon configurations were evaluated and showed the presence of a spinodal in Fe-C up to temperatures near $1200 \mathrm{~K}$ across a wide range of carbon content, $0<w_{\mathrm{C}}<0.17$. The model assumes a perfect substitutional lattice, where all sites are occupied by iron atoms. Nevertheless, taking into consideration the recent literature, it is concluded that spinodal decomposition is becoming less favorable in the presence of defects.

\section{ACKNOWLEDGMENTS}

The research leading to these results has received funding from the European Research Council under the European Union's Seventh Framework Programme FP7/2007-2013/ERC Grant Agreement Number 306292 .

\section{OPEN ACCESS}

This article is distributed under the terms of the Creative Commons Attribution 4.0 International License (http://creativecommons.org/licenses/by/4.0/), which permits unrestricted use, distribution, and reproduction in any medium, provided you give appropriate credit to the original author(s) and the source, provide a link to the Creative Commons license, and indicate if changes were made.

\section{REFERENCES}

1. Y. Ohmori and I. Tamura: Metall. Trans. A., 1992, vol. 23A, pp. 2147-58.

2. K. Taylor, L. Chang, G. Olson, G. Smith, M. Cohen, and J. vander Sande: Metall. Trans. A, 1989, vol. 20A, pp. 2717-37.

3. B. Kim, J. Sietsma, and M.J. Santofimia: In Proceedings of the International Conference on Solid-Solid Phase Transformations in Inorganic Materials, 2015, pp. 301-02.

4. B. Kim, J. Sietsma, and M.J. Santofimia: Philos. Mag., 2016, vol. 96 , pp. 2632-48.

5. D. Kalish and M. Cohen: Mater. Sci. Eng, 1970, vol. 6, pp. 156-66.

6. R. Veiga, M. Perez, C. Becquart, E. Clouet, and C. Domain: Acta Mater., 2011, vol. 59, pp. 6963-74.

7. F. Danoix, H. Zapolsky, S. Allain, and M. Goune: In Proceedings of the International Conference on Solid-Solid Phase Transformations in Inorganic Materials, 2015, pp. 537-38.

8. M. Herbig, R.K.W. Marceau, L. Morsdorf, and D. Raabe: In Proceedings of the International Conference on Solid-Solid Phase Transformations in Inorganic Materials, 2015, pp. 537-38.

9. S. Allain, F. Danoix, M. Goune, K. Hoummada, and D. Mangelinck: Philos. Mag. Lett., 2013, vol. 93, pp. 68-76.

10. S.B. Ren and S.T. Wang: Metall. Trans. A., 1988, vol. 19A, pp. 2427-32.

11. S.B. Ren, T. Tadaki, K. Shimizu, and X.T. Wang: Metall. Mater. Trans. A, 1995, vol. 26A, pp. 2001-05.

12. R. Naraghi, M. Selleby, and J. Ågren: CALPHAD, 2014, vol. 46, pp. 148-58.

13. M. Hillert: Phase Equilibria, Phase Diagrams and Phase Transformations: Their Thermodynamic Basis, 2nd ed., University Press, Cambridge, 2008.

14. D.A. Porter, K.E. Easterling, and M.Y. Sherif: Phase Transformations in Metal and Alloys, 3rd ed., CRC Press, Boca Raton, 2009.

15. C. Zener: Trans. AIME, 1946, vol. 167, pp. 550-95.

16. M. Hillert: Z. Metallkd., 1999, vol. 90, pp. 60-63.

17. C. Domain, C.S. Becquart, and J. Foct: Phys. Rev. B., 2004, vol. 69 (144112), pp. 1-16.

18. K. Taylor and M. Cohen: Prog. Mater. Sci., 1992, vol. 36, pp. 225-72.

19. D.E. Jiang and E.A. Carter: Phys. Rev. B, 2003, vol. 67 (214103), pp. $1-11$.

20. J.H. Jang, H.K.D.H. Bhadeshia, and D.W. Suh: Scripta Mater., 2013, vol. 68, pp. 195-98.

21. C. Först, J. Slycke, K.J. Van Vliet, and S. Yip: Phys. Rev. Lett., 2006, vol. 96 (175501), pp. 1-4.

22. M. Souissi, Y. Chen, M. Sluiter, and H. Numakura: Comput. Mater. Sci., 2016, vol. 124, pp. 249-58.

23. J. Hilliard: In Phase transformations: papers presented at a seminar of the American Society for Metals. American Society for Metals, 1970 , p. 518

24. L. Cheng, A. Böttger, T. de Keijser, and E. Mittemeijer: Scripta Metall. Mater., 1990, vol. 24, pp. 509-14.

25. J.W. Cahn: Acta Metall., 1962, vol. 10, pp. 179-83.

26. S.M. Kim and W.J.L. Buyers: J. Phys. F, 1978, vol. 8, pp. 103-08.

27. R. McLellan and M. Wasz: Phys. Stat. Sol. A, 1988, vol. 110, pp. 421-27.

28. O. Seydel, G. Frohberg, and H. Wever: Phys. Stat. Sol. A, 1994, vol. 144, pp. 69-79. 